\title{
Comportamento reológico de suco misto elaborado com frutas tropicais
}

\author{
Rheological properties of tropical juices
}

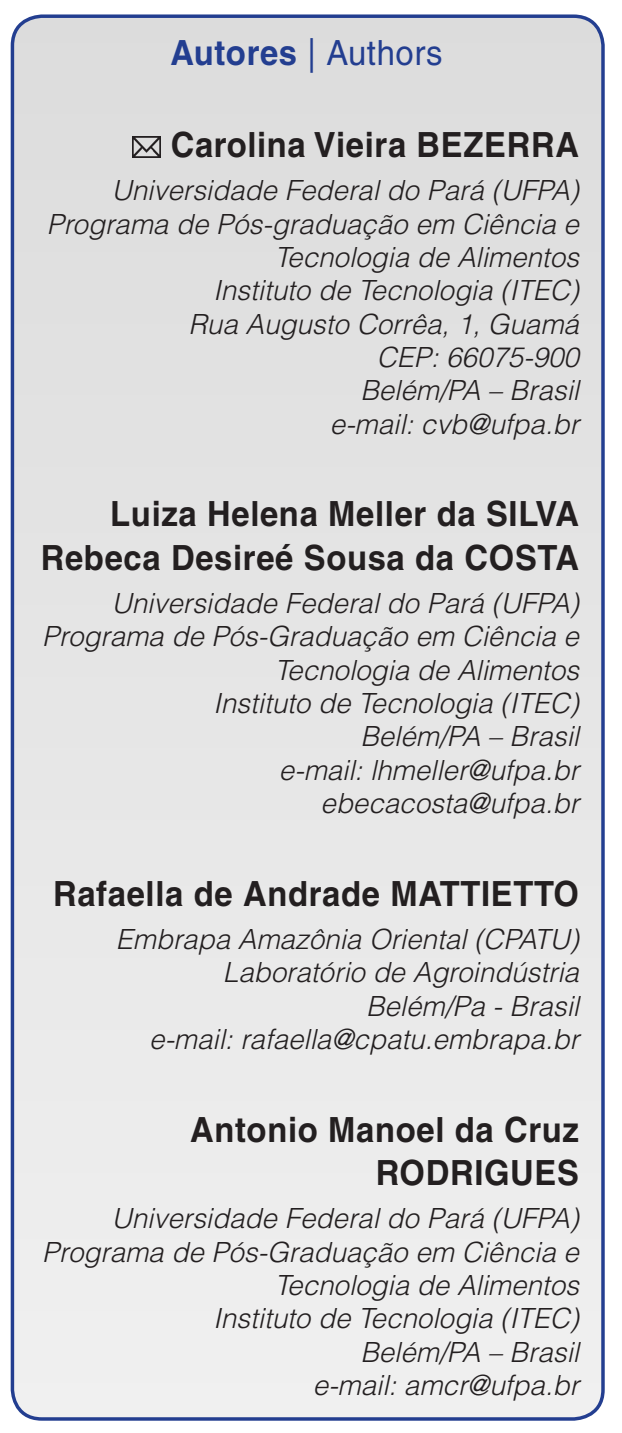

$\triangle$ Autor Correspondente / Corresponding Author

Recebido / Received: 04/09/2012

Aprovado / Approved: 18/04/2013

Publicado / Published: jun./2013

\section{Resumo}

Neste trabalho, foi determinado o comportamento reológico do suco misto obtido a partir de uma mistura de frutas tropicais, mediante um viscosímetro de cilindros concêntricos, no intervalo de temperatura de $10^{\circ} \mathrm{C}$ a $60^{\circ} \mathrm{C}$. Os modelos Lei da Potência e Mizrahi-Berk foram ajustados aos dados experimentais. Os modelos mostraram-se adequados para descrever o comportamento reológico do suco misto, de acordo com os parâmetros estatísticos de ajuste. O produto apresentou índice de comportamento menor do que 1 (um), caracterizando a pseudoplasticidade do suco. Os índices de comportamento de consistência decresceram com o aumento da temperatura. O efeito da temperatura no comportamento reológico do suco foi descrito por uma equação análoga à de Arrhenius e discutida em termos de energia de ativação $\left(E_{a}\right)$. Os valores de $E_{a}$ variaram de 4,27 a 4, $66 \mathrm{kcal}^{\mathrm{gmol}}{ }^{-1}$, com o aumento da taxa de deformação.

Palavras-chave: Reologia; Viscosidade; Arrhenius; Suco misto.

\section{Summary}

The rheological properties of a juice blend obtained from a tropical fruit mix were determined in this work using a rotational viscosimeter at temperatures from $10{ }^{\circ} \mathrm{C}$ to $60{ }^{\circ} \mathrm{C}$. The Power law and Mizrahi-Berk models were fitted to the experimental data. All the models were considered adequate to describe the rheological properties of the juice blend according to the fitted statistical parameters. The behaviour index of the product was below 1.0, indicating the pseudoplasticity of the juice. The behaviour indexes for consistency were found to decrease with increase in temperature. The effect of temperature on the rheological behaviour of the juice was described by an Arrhenius type equation and discussed in terms of the activation energy $\left(E_{a}\right)$. The values for $E_{a}$ varied from 4.27 to $4.66 \mathrm{kcal}$. $\mathrm{gmol}^{-1}$ with increases in shear rate.

Key words: Rheology; Viscosity; Arrhenius; Juice blend. 


\section{Introdução}

Os sucos de frutas tropicais são consumidos e apreciados em todo o mundo não só pelo seu sabor, mas também por serem fontes naturais de carboidratos, carotenoides, vitaminas, minerais e outros componentes importantes. Uma mudança apropriada na dieta em relação à inclusão de componentes encontrados em frutas e suco de frutas pode ser importante na prevenção de doenças e para uma vida mais saudável (BROEK, 1993; SHILS et al., 1994).

O mercado brasileiro de sucos e néctares prontos para beber está em franca expansão, acompanhando a tendência mundial de consumo de bebidas saudáveis, convenientes e saborosas. O processamento da fruta ainda é um nicho de mercado quando comparado a outros destinos da fruta. Segundo a Associação Nacional de Exportação de Sucos Cítricos, em 2008, o setor de sucos, néctares e refrescos de frutas prontas produziu 103.318 milhões de litros; no ano de 2010, essa produção foi de 111.767 milhões de litros. Analisando-se a evolução dos sucos por sabor, o único que teve aumento no período de 2004 a 2010 foi o sabor de "mix" de frutas, passando de 1.502 milhões de litros para 1.582 milhões de litros, respectivamente (CitrusBR, 2010).

No Brasil, preferencialmente os sucos integrais de frutas tropicais são os mais consumidos, com destaque para caju, maracujá, acerola e manga (ESTRELLA, 2004). Atualmente, marcado pelo dinamismo do setor, há um mercado crescente para sucos mistos, sendo que essa tendência é mais observada em produtos que utilizam, em suas formulações, frutas tropicais. Os sucos mistos são sucos elaborados com mais de uma fruta com a finalidade de melhorar as características sensoriais dos componentes isolados, além de poder agregar valor nutricional, seja pelo aumento do teor de vitaminas, seja pela inserção de compostos com características funcionais (MATTIETTO et al., 2006).

Os sucos de frutas, assim como outros produtos derivados de frutas (néctar, polpas concentradas, sorvete), são sistemas bifásicos, compostos por partículas sólidas dispersas em um meio aquoso. Durante seu processamento, estes sistemas alimentares são expostos a vários tipos de tensão, provocada pelo escoamento através de tubulações, bombas, trocadores de calor, misturadores, filtros, entre outros equipamentos de processamento. Estudos demonstram que as condições de processamento têm efeito decisivo na microestrutura e nas propriedades reológicas de sistemas alimentares líquidos (NICKERSON et al., 2003; ALVAREZ et al., 2004; MENG e RAO, 2005; AUGUSTO et al., 2012, 2013)

O conhecimento das características reológicas desempenha papel fundamental no controle de qualidade, no controle do processo e na concepção das linhas de processamento e desenvolvimento de novos produtos (PELEGRINE e GASPARETTO, 2002; COVAS e DIOGO, 2001). Fatores, como concentração de sólidos solúveis, temperatura, distribuição e tamanho de partículas, podem afetar o comportamento reológico de sucos de frutas (RAO e ANANTHESWARAN, 1982; RAO, 1977; VITALI e RAO, 1984; TIZIANI e VODOVOTZ, 2005; TOLSTOGUZOV, 1991). A maioria de alimentos fluidos derivados de frutas apresenta comportamento pseudoplástico, no qual a viscosidade aparente decresce com o aumento da taxa de deformação. Na maioria dos casos, este comportamento não newtoniano pode ser atribuído à presença de substâncias de alto peso molecular em solução e/ou aos sólidos dispersos na fase fluida (SATO e CUNHA, 2007; RAO, 1977)

Considerando-se a crescente necessidade e a procura dos parâmetros reológicos de sucos mistos obtidos a partir de frutas tropicais e levando-se em consideração também que as frutas tropicais originárias da Região Norte brasileira têm sido objeto de pouco estudo de propriedades físicas e reológicas, este trabalho teve como objetivo estudar a influência da temperatura no intervalo de 10 a $60^{\circ} \mathrm{C}$ sobre o comportamento reológico do suco misto elaborado pela combinação de polpas das seguintes frutas: acerola (Malpighia punicifolia L.), maracujá (Passiflora edulis f. flavicarpa) e taperebá (Spondias Lutea L.).

\section{Material e métodos}

\subsection{Matérias-primas}

As polpas de frutas utilizadas neste estudo foram adquiridas no mercado 'Ver-o-peso' localizado na cidade de Belém, Estado do Pará.

\subsection{Preparo do suco misto}

Para a formulação da mistura, utilizaram-se $5 \%$ de polpa de acerola, $10 \%$ de polpa de maracujá e $20 \%$ de polpa de taperebá, adicionados de xarope de sacarose de forma a produzir um produto com teor de sólidos solúveis final de $18^{\circ} \mathrm{Brix}$.

\subsection{Caracterização físico-química do suco misto}

A amostra do suco misto foi caracterizada segundo a metodologia da AOAC (CUNNIFF, 1997), sendo realizadas as seguintes análises: umidade, determinada gravimetricamente conforme o método 16192; proteína, quantificada pelo método Kjeldahl, de acordo com o método 16193; lipídios totais, determinados de acordo com método 948.22, através de um extrator Soxhlet (marca MARCONI, Brasil, modelo MA-487/6/250); cinzas, determinadas pelo método gravimétrico 940.26; carboidratos, calculados pela diferença entre 100 e a 
soma das porcentagens de umidade, proteína, lipídios totais, cinzas e teor de sólidos solúveis ( ${ }^{\circ} \mathrm{Brix}$ ), medidos diretamente em um refratômetro de bancada (QUIMIS, Modelo ABBE Q-109B, Brasil), de acordo com o método 932.12; fibras totais, determinadas pelo método detergente ácido, de acordo com Goering e Vansoest (1970); pH, determinado por meio de leitura direta em potenciômetro (QUIMIS, Modelo Q-400A, Brasil), de acordo com o método 945.10, e acidez titulável, determinada de acordo com a metodologia 942.15, sendo expressa em ácido cítrico.

\subsection{Medidas reológicas}

As propriedades reológicas foram determinadas segundo Vidal et al. (2004), utilizando-se viscosímetro Brookfield (Brookfield Engineering Laboratories, EUA: modelo LVDV-II). Foram utilizados: um Spindle, DIN-85, que permite leitura de taxa de deformação $(\gamma)$ na faixa de 0 a $258 \mathrm{~s}^{-1}$, e o acessório para pequena quantidade de amostra (20 mL), DIN adaptador (Brookfield Engineering Laboratories, EUA). No controle de temperatura, foi empregado um banho termostático Haake B3 (Haake, Karlsruhe, Alemanha). As medidas foram realizadas nas temperaturas de 10, 20, 30, 4050 e $60^{\circ} \mathrm{C}$, por incluírem a temperatura típica de prateleira do produto acabado e a temperatura representativa de pasteurização nas indústrias (FERREIRA et al., 2008). Os dados de

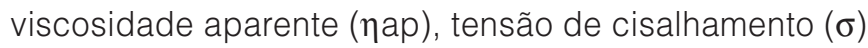
e taxa de deformação $(\gamma)$ foram obtidos utilizando-se o software WinGather ${ }^{\circledR}$ (versão V1.1, Brookfield Engineering Laboratories, EUA). O procedimento de medida para todas as amostras foi realizado em triplicata, sendo obtidos então valores de tensão de cisalhamento relativos às taxas de deformação aplicadas, que variaram na faixa de 0 a $258 \mathrm{~s}^{-1}$, sendo que em cada repetição utilizou-se nova amostra para evitar possíveis efeitos de tempo. O valor final dos parâmetros foi a média resultante das três leituras. O desvio médio global entre as medidas de tensão para o intervalo de temperatura investigado (10-60 ${ }^{\circ} \mathrm{C}$ ), neste trabalho, foi de $\pm 0,03 \mathrm{~Pa}$, variando de 0,01 a $0,05 \mathrm{~Pa}$.

Os modelos Lei da Potência (Equação 1) e MizrahiBerk (Equação 2) foram ajustados aos dados obtidos de tensão de cisalhamento versus taxa de deformação, por meio de regressão não linear com auxílio do software STATISTICA (versão 5.5, Statsoft - USA).

$\tau=\mathrm{k} \gamma^{\mathrm{n}}$

$\tau^{1 / 2}=k_{o M}+k_{M} \gamma^{n}$

em que: $\tau=$ Tensão de cisalhamento $(\mathrm{Pa}) ; \gamma=$ Taxa de deformação $\left(\mathrm{s}^{-1}\right) ; \mathbf{K}_{0 \mathrm{M}}=$ raiz quadrada da tensão inicial de Mizrahi-Berk $\left(\mathrm{Pa}^{1 / 2}\right) ; \mathbf{k}$ e $\mathbf{k}_{\mathbf{M}}=$ Índices de consistência da Lei da Potência $\left(P a . s^{n}\right)$ e de Mizrahi-Berk
$\left(\mathrm{Pa}^{1 / 2} \cdot \mathrm{s}^{\mathrm{n}}\right)$, respectivamente; $\mathbf{n}=$ Índice de comportamento (adimensional).

A análise do ajuste dos modelos da Lei da Potência (LP) e de Mizrahi Berk (MB) foi feita com base nos parâmetros: coeficiente de correlação $R^{2}$ (mede a proporção da variação total da média explicada pela regressão), definido como razão entre a soma quadrática total (Equação 3), pelo qui-quadrado $\left(\chi^{2}\right)$, que expressa a diferença entre valores obtidos experimentalmente (Equação 4), e pela soma dos quadrados dos resíduos (SSR), que identifica o erro no ajuste do modelo (Equação 5).

$R^{2}= \pm \frac{\sum\left(y_{\text {pred }}-\tilde{y}\right)^{2}}{\sum\left(y_{\text {med }}-\tilde{y}\right)^{2}}$

$\chi^{2}=\sum_{i=1}^{n}\left[\frac{\left(y_{\text {med }}-y_{\text {pred }}\right)^{2}}{y_{\text {pred }}}\right]$

$\operatorname{SSR}=\sum_{i=1}^{n}\left(y_{\text {med }}-y_{\text {pred }}\right)^{2}$

em que: $\mathrm{y}_{\text {pred. }}=$ valor previsto pelo modelo; $\mathrm{y}_{\text {med }}=$ valor experimental; $\tilde{y}=$ média amostral.

Quanto maior for o valor de $\chi^{2}$, maior será a discrepância entre as frequências observadas e esperadas. Quanto menor o valor de $\mathrm{R}^{2}$, maior a distância dos pontos experimentais ao modelo ajustado. Portanto, 0 modelo que melhor se ajusta é aquele com altos valores de $R^{2}($ máximo $=1)$ e baixos valores de $\chi^{2}($ mínimo $=0)$.

Em geral, a influência da temperatura sobre a viscosidade aparente $\left(\eta_{a}\right)$ de fluidos alimentícios, a uma taxa de deformação específica, tem sido descrita empregando-se a relação do tipo Arrhenius (Equação 6) (RAO, 1999).

$\eta_{\mathrm{a}}=\eta_{\mathrm{o}} \exp \left(\frac{E_{\mathrm{a}}}{R T}\right)$

em que: $\eta_{a}=$ viscosidade aparente (Pa.s); $\eta_{0}=$ fator pré-exponencial ( $\mathrm{Pa} . \mathrm{s}) ; \mathrm{R}=$ constante universal dos gases $\left(1,987 \times 10^{-3} \mathrm{kcal} \cdot \mathrm{gmol}^{-1} \cdot \mathrm{K}^{-1}\right) ; \mathrm{E}_{\mathrm{a}}=$ energia de ativação para escoamento viscoso $\left(\mathrm{kcal}^{\mathrm{gmol}} \mathrm{g}^{-1}\right) ; \mathrm{T}=$ temperatura absoluta (K).

O valor da energia de ativação foi determinado a partir da variação da viscosidade aparente com a temperatura mediante o ajuste da Equação 6, por meio de regressão linear.

A viscosidade aparente, em função do modelo LP, foi calculada pelos dados de índice de consistência (k), índice de comportamento do fluido (n) e taxa de cisalhamento (Equação 7) que, rearranjada com o modelo LP (Equação 1), resultou na Equação 8. 
$\eta_{\text {ap }}=\frac{\tau}{\gamma}$

$\eta_{\mathrm{ap}}=\mathrm{k}(\gamma)^{(\mathrm{n}-1)}$

\section{Resultados e discussão}

Os resultados da análise físico-química do suco misto estão apresentados na Tabela 1. Os parâmetros 'acidez total' e 'sólidos solúveis' encontram-se dentro dos padrões de identidade e qualidade para sucos tropicais (BRASIL, 2003). A mistura de acerola, maracujá e taperebá pode ser considerada como um produto novo, não tendo referência de comparação dos valores para proteínas, lipídios, cinzas e carboidratos; porém, os valores estão dentro da faixa esperada para produtos à base de frutas. A mistura de acerola, maracujá e taperebá pode agregar valor nutricional ao produto final, sendo esta

Tabela 1. Composição centesimal do suco misto em base úmida (b.u.).

\begin{tabular}{lr}
\multicolumn{1}{c}{ Parâmetros } & \multicolumn{1}{c}{ Média \pm DP } \\
\hline Umidade (\%) & $87,43 \pm 0,12$ \\
Proteína (\%) & $0,28 \pm 0,01$ \\
Lipídios totais (\%) & $0,29 \pm 0,02$ \\
Carboidratos totais (\%) & $11,84 \pm 0,10$ \\
Cinzas (\%) & $0,16 \pm 0,04$ \\
Fibra total (\%) & $0,52 \pm 0,16$ \\
Sólidos solúveis $\left({ }^{\circ}\right.$ Brix a $\left.20{ }^{\circ} \mathrm{C}\right)$ & $18,2 \pm 0,31$ \\
Acidez Total Titulável $(\%$ ác. cítrico) & $0,72 \pm 0,01$ \\
pH & $3,3 \pm 0,01$ \\
\hline
\end{tabular}

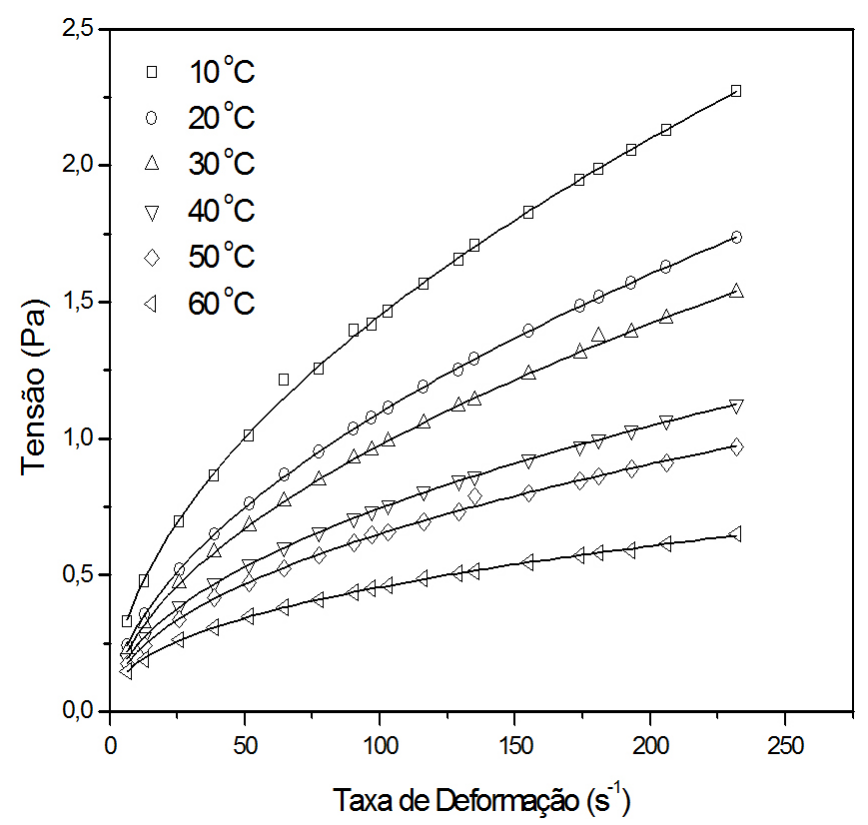

Figura 1. Modelo Lei da Potência ajustado ao comportamento reológico do suco misto. uma estratégia interessante quando se objetiva o caráter funcional. Segundo Mattietto et al. (2006), a mistura de acerola, maracujá e taperebá é uma boa fonte de vitamina $\mathrm{C}$ e é um produto que contribui como um alimento pró-vitamina A, já que as três polpas utilizadas são fontes de carotenoides. Segundo Amaya et al. (2008), o conteúdo de carotenoides da acerola é de $232 \mu \mathrm{g}$ RAE/100 g, sendo a grande parte sob forma de $\beta$-caroteno; no maracujá, o valor médio é de $57 \mu \mathrm{g}$ RAE/100 g, sendo encontrado também sob a forma de $\beta$-caroteno; no taperebá, os valores são de $88 \mu \mathrm{g} R A E / 100 \mathrm{~g}$, sendo encontrado principalmente sob a forma de $\beta$-criptoxantina.

As Figuras 1 e 2 mostram os efeitos da temperatura no comportamento reológico do suco misto; são apresentados os pontos experimentais médios e suas respectivas curvas de ajuste dos modelos LP e MB. Observa-se que, para taxa deformação fixa, a tensão de cisalhamento diminui com o aumento da temperatura. De acordo com Hassan e Hobani (1998), com o aumento da temperatura, a energia térmica e as distâncias moleculares aumentam em função da redução das forças intermoleculares. Associado ao aumento da temperatura, observa-se também que a inclinação das curvas diminui com o aumento da taxa de deformação. Isto evidencia uma diminuição da viscosidade aparente com o aumento da taxa de deformação.

Este comportamento pode ser justificado pela mudança estrutural da amostra decorrente das forças hidrodinâmicas geradas, o que faz com que as partículas se rearranjem em direções paralelas, ocorrendo a quebra em partículas menores. As mesmas podem escoar mais facilmente na direção da tensão aplicada, como um



Figura 2. Modelo Mizrahi-Berk ajustado ao comportamento reológico do suco misto. 
resultado da diminuição da interação partícula-partícula (KESHANI et al., 2012; RAO, 1999; CHIN et al., 2009; SHAMSUDIN et al., 2013).

Os parâmetros relativos aos modelos LP e MB, obtidos por meio de ajuste aos dados experimentais do suco misto, estão nas Tabelas 2 e 3, respectivamente.

Ambos os modelos utilizados para descrever o comportamento reológico do suco misto adequaram-se satisfatoriamente em toda a faixa de temperatura estudada, apresentando baixos valores de SSR e $\chi^{2}$, e valores elevados para o coeficiente de determinação $R^{2}$, superiores a 0,99 . Verifica-se que o índice de comportamento (n) em todas as temperaturas analisadas apresentou valores inferiores a um (1), indicando um comportamento não newtoniano e tipicamente pseudoplástico para as amostras de suco misto. Comportamento semelhante foi observado em outros estudos de caracterização reológica de fluidos derivados de frutas: Silva et al. (2005), estudando suco de acerola; Pelegrine e Gaspareto (2002), avaliando o comportamento reológico de polpa de manga e abacaxi, e Dak et al. (2007), avaliando suco de manga.

Com o aumento da temperatura, observou-se uma diminuição no índice de comportamento (n) em ambos os modelos utilizados. Essa tendência é bem definida para o modelo MB, em toda a faixa de temperatura analisada, com um decréscimo de 78,40 \% entre as temperaturas de 10 e $60^{\circ} \mathrm{C}$. Para o modelo LP, essa tendência se estabelece a partir da temperatura de $20^{\circ} \mathrm{C}$, com um decréscimo de $77,44 \%$ entre as temperaturas de 10 e $60{ }^{\circ} \mathrm{C}$.

Verifica-se também um decréscimo nos valores dos parâmetros $\mathbf{k}$ e $\mathbf{k}_{\mathbf{M}}$ dos modelos LP e MB, respectivamente, com o aumento da temperatura. Comportamento semelhante foi descrito por Vidal et al. (2004), analisando o efeito da temperatura no comportamento reológico da polpa de manga,

Na Figura 3, apresenta-se a relação entre viscosidade aparente $\left(\eta_{\mathrm{ap}}\right)$ e a taxa de deformação para o suco misto. Conforme esperado, verifica-se o decréscimo da viscosidade aparente, considerando-se isoladamente os aumentos de temperatura e taxa de deformação.

Observa-se, na Figura 3, a formação de duas regiões bem distintas, às quais correspondem comportamentos estruturais bem diferenciados. A primeira região, zona de taxa de deformação inferior a $80 \mathrm{~s}^{-1}$, na qual as forças de agregação, em razão do movimento browniano, começam a ser superadas pelas forças de cisalhamento, induzindo o alinhamento das partículas, de modo que a viscosidade aparente do suco misto decresce exponencialmente. $\mathrm{Na}$ segunda região, zona de taxa de deformação superior a $80 \mathrm{~s}^{-1}$, o estado de alinhamento e orientação das partículas encontra-se plenamente estabelecido, e a viscosidade aparente do suco tende a ficar constante, indicando um comportamento newtoniano para o suco. Comportamento semelhante foi observado em outros estudos de caracterização reológica de fluidos derivados de frutas (HAMINIUK et al., 2006; SILVA et al., 2005).

Do ponto de vista industrial, a diminuição da viscosidade aparente facilita o escoamento da polpa e a troca de calor durante o processamento. Quanto menor a viscosidade de um fluido, menor será a perda de carga durante o escoamento.

A equação de Arrhenius (Equação 6) apresentou uma boa descrição do efeito da temperatura na viscosidade aparente do suco misto, nas taxas de deformação utilizadas, como pode ser visto na Figura $4 \mathrm{e}$ na Tabela 4. A variação em torno da média $\left(R^{2}\right)$ apresentou

Tabela 2. Parâmetros do ajuste do modelo Lei da Potência para o suco misto.

\begin{tabular}{lcccccc}
\multicolumn{1}{r}{ Parâmetros } & $\mathbf{1 0}$ & $\mathbf{2 0}$ & $\mathbf{3 0}$ & $\mathbf{4 0}$ & $\mathbf{5 0}$ & $\mathbf{6 0}$ \\
$\mathrm{k}\left(\mathrm{Pa} . \mathrm{S}^{\mathrm{n}}\right)$ & 0,125 & 0,087 & 0,081 & 0,078 & 0,069 & 0,068 \\
$\mathrm{n}$ & 0,532 & 0,548 & 0,539 & 0,490 & 0,487 & 0,412 \\
$\chi^{2}$ & $3,26 \times 10^{-4}$ & $6,57 \times 10^{-6}$ & $3,66 \times 10^{-6}$ & $1,77 \times 10^{-6}$ & $1,13 \times 10^{-4}$ & $3,69 \times 10^{-6}$ \\
$\mathrm{SSR}$ & 0,0055 & $1,11 \times 10^{-4}$ & $4,39 \times 10^{-5}$ & $2,13 \times 10^{-5}$ & 0,0013 & $4,43 \times 10^{-5}$ \\
$\mathrm{R}^{2}$ & 0,9995 & 0,9998 & 0,9998 & 0,9998 & 0,9973 & 0,9997 \\
\hline
\end{tabular}

Tabela 3. Parâmetros do ajuste do modelo Mizrahi-Berk para o suco misto.

\begin{tabular}{lccccccc}
\multicolumn{1}{c}{ Parâmetros } & $\mathbf{1 0}$ & $\mathbf{2 0}$ & $\mathbf{3 0}$ & $\mathbf{4 0}$ & $\mathbf{5 0}$ & $\mathbf{6 0}$ \\
$\mathrm{K}_{\mathrm{OM}}\left(\mathrm{Pa}^{1 / 2}\right)$ & 0,221 & 0,145 & 0,129 & 0,127 & 0,114 & 0,118 \\
$\mathrm{~K}_{\mathrm{M}}\left(\mathrm{Pa}^{1 / 2} \cdot \mathrm{S}^{\mathrm{n}}\right)$ & 0,221 & 0,203 & 0,200 & 0,192 & 0,189 & 0,171 \\
$\mathrm{n}$ & 0,324 & 0,323 & 0,315 & 0,291 & 0,282 & 0,254 \\
$\chi^{2}$ & $8,55 \times 10^{-4}$ & $9,40 \times 10^{-5}$ & $1,08 \times 10^{-4}$ & $3,82 \times 10^{-5}$ & $1,39 \times 10^{-4}$ & $2,0 \times 10^{-5}$ \\
$\mathrm{SSR}$ & 0,014 & 0,002 & 0,002 & $6,11 \times 10^{-4}$ & 0,002 & $3,2 \times 10^{-4}$ \\
$\mathrm{R}^{2}$ & 0,9976 & 0,9996 & 0,9994 & 0,9995 & 0,9977 & 0,9991 \\
\hline
\end{tabular}




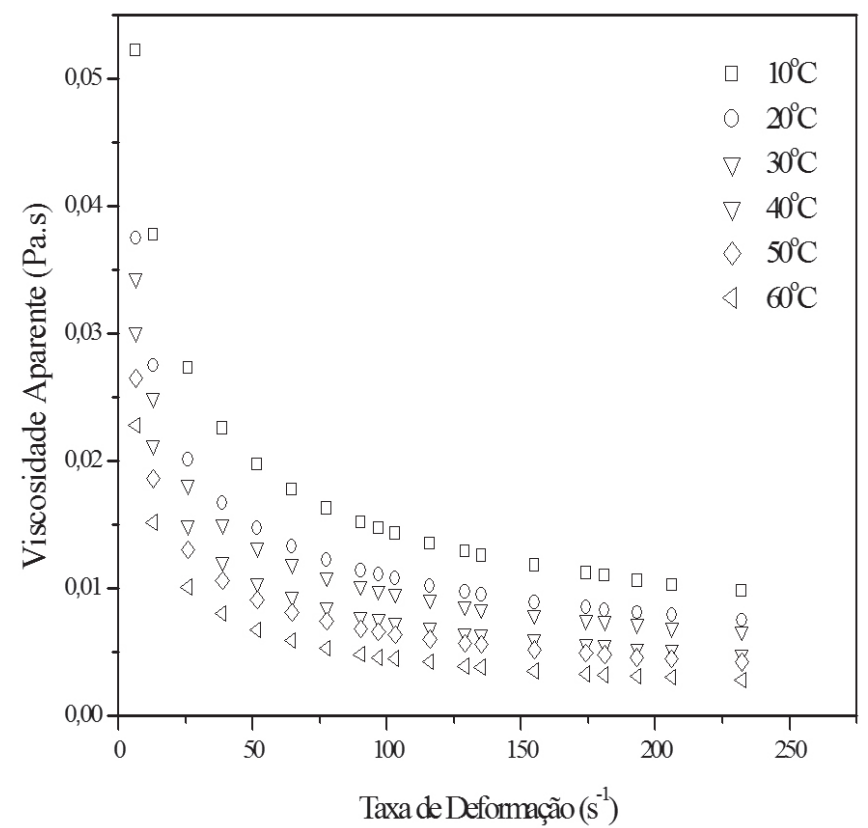

Figura 3. Curvas da viscosidade aparente do suco misto calculadas pelo modelo Lei da Potência.

Tabela 4. Parâmetros da equação de Arrhenius para diferentes taxas de deformação.

\begin{tabular}{cccc}
$\begin{array}{c}\text { Taxa de Deformação } \\
\left(\mathbf{s}^{-1}\right)\end{array}$ & $\begin{array}{c}\eta_{\mathbf{o}} \\
(\mathbf{m P a} \mathbf{s})\end{array}$ & $\begin{array}{c}\mathbf{E}_{\mathbf{a}} \\
(\mathbf{k c a l . g m o l - 1 )}\end{array}$ & $\mathbf{R}^{\mathbf{2}}$ \\
\hline 100 & $7,67 \times 10^{-3}$ & 4,27 & 0,9845 \\
150 & $4,50 \times 10^{-3}$ & 4,48 & 0,9799 \\
200 & $2,91 \times 10^{-3}$ & 4,66 & 0,9746 \\
\hline
\end{tabular}

valores superiores a 0,97 . Os valores da energia de ativação ( $\left.E_{a}\right)$ obtidos neste trabalho são compatíveis aos encontrados na literatura para sucos obtidos a partir de frutas tropicais. Na medida em que a energia de ativação está relacionada com a interação entre as moléculas, os valores encontrados para Ea podem indicar se a viscosidade do produto é mais ou menos influenciada pela variação de temperatura. Silva et al. (2005), ao analisarem o comportamento reológico do suco de acerola, obtiveram valores $\mathrm{E}_{\mathrm{a}}$ variando de 1,79 a 3,50 $\mathrm{kcal} \mathrm{gmol}^{-1}$. Bonomo et al. (2009), estudando o comportamento reológico de sucos de caju, encontraram valores de $E_{a}$ na faixa de 2,99 a 4,46 kcal.gmol-1. Observa-se o aumento da $\mathrm{E}_{\mathrm{a}}$ com a elevação da taxa de deformação.

\section{Conclusões}

O suco misto apresentou, na faixa de temperatura estudada, um comportamento peculiar. Observou-se a formação de duas regiões bem distintas, às quais correspondem a comportamentos estruturais bem diferenciados. Na primeira região, zona de taxa de deformação inferior a $80 \mathrm{~s}^{-1}$, a viscosidade aparente do

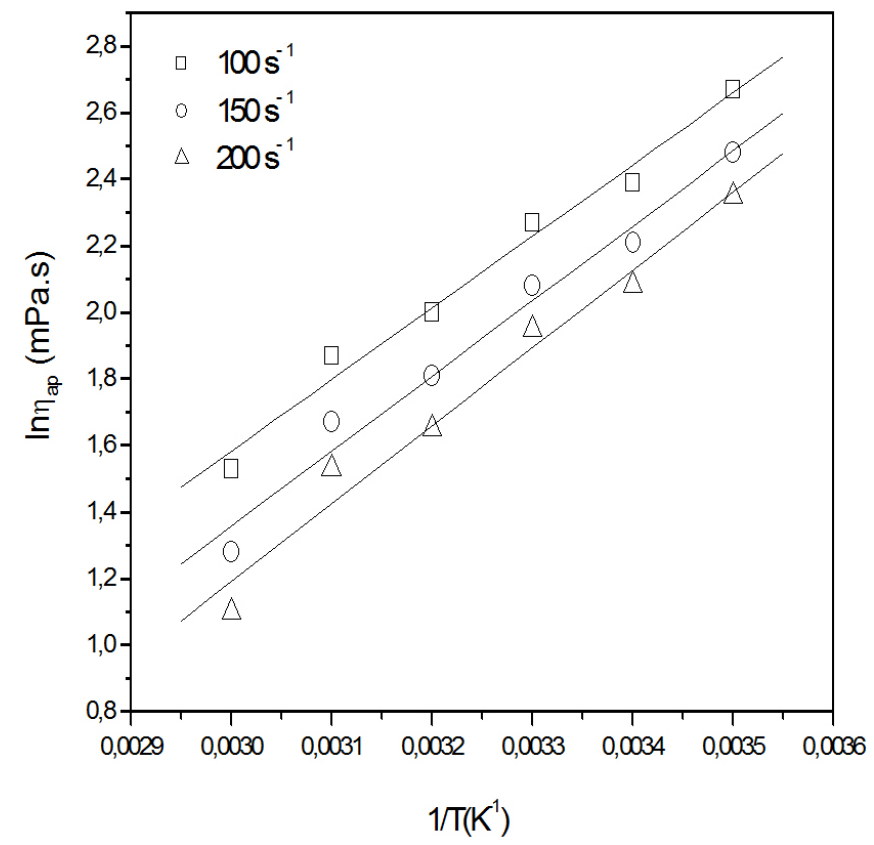

Figura 4. Efeito da temperatura sobre a viscosidade aparente do suco misto ajustado pela equação de Arrhenius.

suco misto decresceu exponencialmente; na segunda região, zona de taxa de deformação superior a $80 \mathrm{~s}^{-1}$, a viscosidade aparente do suco tendeu a ficar constante, indicando um comportamento newtoniano para o suco.

Os índices de comportamento do suco misto foram inferiores a 1 (um), o que caracteriza o comportamento pseudoplástico. O modelo LP mostrou-se mais interessante para simular o comportamento reológico do suco misto, pois, nas condições estudadas, apresentou valores de $\mathrm{R}^{2}$ superiores aos encontrados para o modelo MB. A equação de Arrhenius representou bem o efeito da temperatura na viscosidade aparente do suco misto, apresentando valores de $\mathrm{R}^{2}$ superiores a 0,98. Os valores

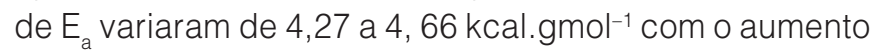
da taxa de deformação.

\section{Referências}

ALVAREZ, M. D.; FERNÁNDEZ, C.; CANET, W. Rheological behaviour of fresh and frozen potato puree in steady and dynamic shear at different temperatures. European Food Research Technology, Berlin, v. 218, n. 6, p. 544-553, 2004. http://dx.doi.org/10.1007/s00217-004-0897-2

AMAYA, D. B. R.; KIMURA, M.; FARFAN, J. A. Fontes Brasileiras de Carotenóides. Brasilia: MMA/SBF, 2008. 100 p.

CUNNIFF, P. (Ed.). Official Methods of Analysis of the Association of Official Analytical Chemists. 16th ed., 3rd rev. Gaithersburg: AOAC, 1997. v. 1.

ASSOCIAÇÃO NACIONAL DE EXPORTADORES DE SUCOS CITRICOS - CitrusBR. Resumo do Consumo Mundial 
Comportamento reológico de suco misto elaborado com frutas tropicais

BEZERRA, C. V. et al.

de Bebidas: Segmentação de Sucos 2004-2010. São Paulo: CitrusBR, 2010. Disponível em: <http://http://www. citrusbr.com/exportadores-citricos/consumo/estatisticas-deconsumo-228460-1.asp >. Acesso em: 23 ago. 2012.

AUGusto, P. E. D.; IBARZ, A.; CRISTIANINI, M. Effect of temperature on dynamic and steady-state shear rheological properties of siriguela (Spondias purpurea L.) pulp. Journal of Food Engineering, Oxford, v. 108, n. 2, p. 283-289, 2012. http:// dx.doi.org/10.1016/j.jfoodeng.2011.08.015

AUGUSTO, P. E. D.; IBARZ, A.; CRISTIANINI, M. Effect of high pressure homogenization $(\mathrm{HPH})$ on the rheological properties of tomato juice: viscoelastic properties and the Cox-Merzrule. Journal of Food Engineering, Oxford, v. 114, n. 1, p. 57-63, 2013. http://dx.doi.org/10.1016/j.jfoodeng.2012.07.025

BONOMO, R. C. F.; FONTAN, R. C. I.; SOUZA, T. S.; VELOSO, C. M.; CASTRO, S. S. Thermophysical properties of cashew juice at different concentrations and temperatures. Revista Brasileira de Produtos Agroindustriais, Campina Grande, v. 11, n. 1, p. $35-42,2009$.

BRASIL. Ministério da Saúde. Agência Nacional de Vigilância Sanitária. Instrução normativa $n^{\circ}$ 12, de 4 de setembro de 2003. Aprova o Regulamento Técnico para Fixação dos Padrões de Identidade e Qualidade Gerais para Suco Tropical; os Padrões de Identidade e Qualidade dos Sucos Tropicais de Abacaxi, Acerola, Cajá, Caju, Goiaba, Graviola, Mamão, Manga, Mangaba, Maracujá e Pitanga; e os Padrões de Identidade e Qualidade dos Néctares de Abacaxi, Acerola, Cajá, Caju, Goiaba, Graviola, Mamão, Manga, Maracujá, Pêssego e Pitanga. Diário Oficial da República Federativa do Brasil, Brasília, DF, 09 set. 2003. Seção 1, n. 174.

BROEK, A. V. D. Functional Foods. The Japanese Approach. International Food Ingredients, Warsal, n. 1-2. p. 4-10, 1993.

CHIN, N. L.; CHAN, S. L.; YUSOF, Y. A.; CHUAH, T. G.;TALIB, R. A. Modelling of rheological of pummelo juice concentrates using master-curve. Journal of Food Engineering, Oxford, v. 93, n. 1, p. 134-140, 2009. http://dx.doi.org/10.1016/j. jfoodeng.2009.01.005

COVAS, J. A.; DIOGO, A. C. A Reologia e as suas Aplicações Industriais. Lisboa: Castro, 2001. 135 p.

DAK, M.; VERMA, R. C.; JAAFFREY, S. N. A. Effect of temperature and concentration on rheological properties of "Kesar" mango juice. Journal of Food Engineering, Oxford, v. 80 , n. 4, p. 1011-1015, 2007. http://dx.doi.org/10.1016/j. jfoodeng.2006.08.011

ESTRELLA, A. Sucos tropicais no Brasil para consumo local. In: JUICE LATIN AMERICA, 2004, São Paulo. Anais... São Paulo: [s.n.], 2004. CD-ROM.

FERREIRA, G. M.; GUIMARÃES, M. J. O. C.; MAIA, M. C. A. Efeito da temperatura e taxa de cisalhamento nas propriedades de escoamento da polpa de cupuaçu (Theobroma grandiflorum Schum) integral. Revista Brasileira Fruticultura, Jaboticabal, v. 30, n. 2, p. 385-389, 2008.

GOERING, H. K.; VANSOEST, P. J. Forage Fibre Analysis: Apparatus, Reagents, Procedures and Some Applications. Washington: USA, 1970. p. 375.

HAMINIUK, C. W. I.; SIERAKOWSKI, M. R.; VIDAL, J. R. M. B.; MASSON, M. L. Influence of temperature on the rheological behavior of whole araçá pulp (Psidium cattleianum sabine). LWT - Food Science and Technology, London, v. 39, n. 4, p. 427-431, 2006

HASSAN, B. H.; HOBANI, A. I. Flow properties of roselle (Hibiscus sabdariffa L.) extract. Journal of Food Engineering, Oxford, v. 35, n. 4, p. 459-470, 1998. http://dx.doi.org/10.1016/ S0260-8774(98)00044-2

KESHANI, S.; LUQMAN, A. C.; RUSSLY, A. R. Effect of temperature and concentration on rheological properties pomelo juice concentrates. International Food Research Journal, Selangor, v. 19, n. 2, p. 553-562, 2012.

MATtietto, R. A.; YANO, Y. B.; VASCONCELOS, M. A. M. Caracterização de um "Blend"Tropical Elaborado com Polpa de Maracujá, Acerola e Taperebá. Manaus: Embrapa Amazônia Oriental, 2006. 17 p. (Boletim de Pesquisa e Desenvolvimento, ก. 59).

MENG, Y; RAO, M. A. Rheological and structural properties of cold-water-swelling and heated cross-linked waxy maize starch dispersions prepared in apple juice and water. Carbohydrate Polymers, Norwich, v. 60, n. 3, p. 291-300, 2005. http://dx.doi. org/10.1016/j.carbpol.2005.01.012

NICKERSON, M. T.; PAULSON, A. T.; SPEERS, R. A. Rheological properties of gellan solutions: effect of calcium ions and temperature on pre-gel formation. Food Hydrocolloids, Oxford, v. 17, n. 5, p. 577-583, 2003. http://dx.doi.org/10.1016/S0268$005 \times(02) 00075-9$

PELEGRINE, D. H.; GASPARETTO, C. A. Rheological behavior of pineapple and mango pulps. Lebensmittel-Wissenschaft und Technologie Food Science and Technology, Berlin, v. 35, p. 645-648, 2002.

RAO, M. A. Rheology of Fluid and Semisolid Foods: Principle and Applications. Gaithersburg: Aspen Publishers Inc, 1999. $482 \mathrm{p}$.

RAO, M. A. Rheology of liquid foods - a review. Journal of Texture Studies, Westport, v. 8, p. 135-168, 1977. http://dx.doi. org/10.1111/j.1745-4603.1977.tb01173.x

RAO, M. A.; ANANTHESWARAN, R. C. Rheology of fluids in food processing. Food Technology, Chicago, v. 36, n. 2, p. 116-126, 1982. 
Silva, F. C.; Guimarães, D. H. P.; Gasparetto, C. A. Reologia do suco de acerola: efeitos da concentração e temperatura. Ciência e Tecnologia de Alimentos, Campinas, v. 25, n. 1, p. 121-126, 2005. http://dx.doi.org/10.1590/S010120612005000100020

SATO, A. C. K.; CUNHA, R. L. Influência da temperatura no comportamento reológico da polpa de jabuticaba. Ciência e Tecnologia de Alimentos, Campinas, v. 27 , n. 4, p. 890-896, 2007. http://dx.doi.org/10.1590/S010120612007000400033

SHAMSUDIN, R.; LING, C. S.; ADZAHAN, N. M.; DAUD, W. R. W. Rheological properties of ultraviolet-irradiated and thermally pasteurized Yankee pineapple juice. Journal of Food Engineering, Oxford , v. 116, n. 2, p. 548-553, 2013. http:// dx.doi.org/10.1016/j.jfoodeng.2012.12.031

SHILS, M. E.; OLSON, J. A.; SHIKE, M. Modern Nutrition in Health and Disease. 8th ed. Philadelphia: Lea Febiger, 1994. 1951p.
TIZIANI, S.; VODOVOTZ, Y. Rheological Effects of soy protein addition on tomate juice. Food Hidrocolloids, Oxford, v. 19, n. 1, p. 45-52, 2005. http://dx.doi.org/10.1016/j.foodhyd.2004.04.012 TOLSTOGUZOV, V. B. Functional properties of food proteins and role of protein-polysaccharide interaction - review. Food Hydrocolloids, Oxford, v. 4, n. 5, p. 429-468, 1991. http://dx.doi. org/10.1016/S0268-005X(09)80196-3

VIDAL, J. R. M. B.; PELEGRINE, D. H.; GASPARETTO, C. A. Efeito da temperatura no comportamento reológico da polpa de manga (mangífera indica L-Keitt). Ciência e Tecnologia de Alimentos, Campinas, v. 24, n. 1, p. 39-42, 2004. http://dx.doi. org/10.1590/S0101-20612004000100008

VITALI, A. A.; RAO, M. A. Flow properties of low-pulp concentrated orange juice: effect of temperature and concentration. Journal of Food Science, Chicago, v. 49, n. 3, p. 882-888, 1984. http:// dx.doi.org/10.1111/j.1365-2621.1984.tb13233.x 